J. Korean Math. Soc. 46 (2009), No. 4, pp. 675-689

DOI 10.4134/JKMS.2009.46.4.675

\title{
THE GROUP OF UNITS OF SOME FINITE LOCAL RINGS III
}

\author{
Sung SiK Woo
}

\begin{abstract}
As a sequel to the papers [2,3], we will complete our identification of the groups of units of the finite local rings $\mathbb{Z}_{4}[X] /\left(X^{k}+\right.$ $\left.2 t(X), 2 X^{r}\right)$ which is the most general type of finite local rings with a single nilpotent generator over $\mathbb{Z}_{4}$.
\end{abstract}

\section{Introduction}

Consider the ring $R=\mathbb{Z}_{4}[X] /\left(X^{k}+2 u(X) X^{a}, 2 X^{r}\right)$ where $u(X)=\sum_{i=0}^{s} a_{i} X^{i}$ with $u(0)=1$, and $\operatorname{deg}(u)<k-a$. We will adopt the convention that $X^{-\infty}=0$ so that our ring $R$ can be $R=\mathbb{Z}_{4}[X] /\left(X^{k}\right)$ if $a=r=-\infty$; or $R=\mathbb{Z}_{4}[X] /\left(X^{k}+\right.$ $\left.2 X^{a}\right)$ if $r=-\infty$. If $a>0$, then the elements of the form $1+X f(X)$, where $f \in \mathbb{Z}_{4}[X]$ form a subgroup of $U(R)$ of the group of units which we denote by $U_{1}(R)$ and we call such an element a 1-unit. In [2, XVIII.2] the group $U_{1}(R)$ is called the one group of $R$. If $a=0$, then the set of 1-units do not form a subgroup and in that case we will consider the group of units $U(R)$ of $R$.

In [2] any finite $\mathbb{Z}_{4}$-algebra which is generated by a single element is of the form $R=\mathbb{Z}_{4}[X] /\left(X^{k}+2 u(X) X^{a}, 2 X^{r}\right)$ with $a<r<k$ and a polynomial $u(X)$ such that $u(0)=1$ and $\operatorname{deg}(u)<r-a$.

In this paper, we will identify the group of units $U(R)$ of $R$ which is a finite abelian 2-group by decomposing into a direct sum of cyclic subgroups thereby completing the identification of the groups of units of the finite rings which is generated by a single nilpotent element. For this we need to find the "natural" generators of the cyclic subgroups.

As in [3], there is a natural surjective ring homomorphism

$$
\phi: \mathbb{Z}_{4}[X] /\left(X^{k}+2 u(X) X^{a}, 2 X^{r}\right) \rightarrow \mathbb{F}_{2}[X] /\left(X^{k}\right)
$$

which induces a surjective group homomorphism which we still call $\phi$,

$$
\phi: U\left(\mathbb{Z}_{4}[X] /\left(X^{k}+2 u(X) X^{a}, 2 X^{r}\right)\right) \rightarrow U\left(\mathbb{F}_{2}[X] /\left(X^{k}\right)\right)
$$

Received August 19, 2007.

2000 Mathematics Subject Classification. 13C12.

Key words and phrases. finite local ring, group of units. 
on the groups of units with $\operatorname{ker}(\phi)=T_{0}$. Hence we have an exact sequence

$$
(1) \rightarrow T_{0} \rightarrow U\left(\mathbb{Z}_{4}[X] /\left(X^{k}+2 u X^{a}, 2 X^{r}\right)\right) \stackrel{\phi}{\rightarrow} U\left(\mathbb{F}_{2}[X] /\left(X^{k}\right)\right) \rightarrow(1),
$$

where $T_{0}$ is generated by $\Delta=\left\{-1+2 X^{i} \mid 0 \leq i<n\right\}$ with $n=k$ if $r=$ $-\infty$; and $n=r$ if $r>0$. Note that we can choose the generators of $T_{0}$ by $\{-1\} \cup\left\{1+2 X^{i} \mid 0<i<n\right\}$.

When $a>0$, we can construct a similar exact sequence for 1-units

$$
(1) \rightarrow T \rightarrow U_{1}\left(\mathbb{Z}_{4}[X] /\left(X^{k}+2 u X^{a}, 2 X^{r}\right)\right) \stackrel{\phi_{1}}{\rightarrow} U\left(\mathbb{F}_{2}[X] /\left(X^{k}\right)\right) \rightarrow(1),
$$

where $T=\operatorname{ker}\left(\phi_{1}\right)$ which is generated by $\Delta_{1}=\left\{1+2 X^{i} \mid 0<i<n\right\}$ with $n=k$ if $r=-\infty$; and $n=r$ if $r>0$.

We let

$$
\begin{aligned}
& G_{i}=\left\langle 1+X^{i}\right\rangle \text { where } i \text { is odd with } 0<i<k, \\
& H_{j}=\left\langle 1+2 X^{j}\right\rangle \text { where } j \text { is an integer with } 0<j<k \text { and } \\
& H_{0}=\langle-1\rangle
\end{aligned}
$$

and let

$$
G=\sum_{1 \leq i: \text { odd }<k} G_{i}, H_{(l)}=\sum_{0<i<l} H_{i} \text { and } H_{(l)}^{+}=\sum_{0 \leq i<l} H_{i}
$$

Also note that if $U_{1}(R)$ is well defined, then $U(R)=H_{0} \oplus U_{1}(R)$ and $T_{0}=$ $H_{0} \oplus T$.

In Section 2, we consider the rings of the form $R=\mathbb{Z}_{4}[X] /\left(X^{k}+2 u(X) X^{a}\right)$ and find a direct sum decomposition of the group of units $U(R)$ of $R$ into a sum of cyclic subgroups. As we pointed out in [3, Proposition 2.6] the generators for the group of units of the $\operatorname{ring} R=\mathbb{Z}_{4}[X] /\left(X^{k}+2 X^{a}\right)$ do not give rise to a direct sum decomposition. It turns out that we need to modify the generators for the group of units of the ring $R=\mathbb{Z}_{4}[X] /\left(X^{k}+2 X^{a}\right)$ to get the generators of the group of units of the ring $R=\mathbb{Z}_{4}[X] /\left(X^{k}+2 u(X) X^{a}\right)$.

In Section 3, we consider the ring $R=\mathbb{Z}_{4}[X] /\left(X^{k}+2 u(X) X^{a}, 2 X^{r}\right)$ and we identify the group of units. When $u=1$ a complete description of the group of units of $R$ as a direct sum of cyclic groups is given in [3].

We will maintain our notations of $[2,3]$ which we recall briefly: For a finite set $S$ of positive integers and a nonnegative integer $\alpha$ we will write $S+\alpha=$ $\{i+\alpha \mid i \in S\}$. And $X^{S}=\sum_{i \in S} X^{i}$ as an element of $\mathbb{Z}_{4}[X]$. If $S=\emptyset$, then we define $X^{S}=0$.

For a rational number $r$ let $\lfloor r\rfloor_{2}$ to be the smallest integer greater than or equal to $\log _{2}(a)$. Hence $2^{\lfloor r\rfloor_{2}}$ is the smallest 2-power which is greater than or equal to $r$. If the order $o(G)$ of a group $G$ is $2^{n}$, then we will say the 2 logarithmic order of $G$ is $n$ and we will write $l_{2}(G)=n$ or simply $l o(G)=n$. For $x \in G$ we will write $l o_{2}(x)$ for the 2-logarithmic order of the subgroup generated by $x$. 


\section{The group of units of the ring $R=\mathbb{Z}_{4}[X] /\left(X^{k}+2 u X^{a}\right)$}

In this section we consider the group of units of the ring $R=\mathbb{Z}_{4}[X] /\left(X^{k}+\right.$ $\left.2 u(X) X^{a}\right)$, where $u(X)=1+X^{b_{1}}+\cdots+X^{b_{s}}$. Also write $t(X)=u(X) X^{a}=$ $X^{a_{0}}+\cdots+X^{a_{s}}$ with $a_{0}<\cdots<a_{s}<k$ and $a_{0}=a$.

Now we look closely at the group structure of $T$. Let $\mathfrak{A}=\{i \mid 0<i<$ $n\}$, where $n=k$ when $r=-\infty$; and $n=r$ when $r>0$. First note that $\left(1+2 X^{i}\right)\left(1+2 X^{j}\right)=1+2 X^{i}+2 X^{j}$. Hence we can identify the multiplicative group $T$ with the additive group

$$
T^{0}=\left\{2 X^{S} \in \mathbb{Z}_{4}[X] \mid S \subset \mathfrak{A}\right\}
$$

which also can be identified with $2 \mathbb{Z}_{4}^{n-1}$. For a polynomial $f(X)=1+2 X^{S}$ we let $f^{0}(X)=f(X)-1=2 X^{S}$.

As in [3], we will make use of the following simple observation.

Lemma 2.1. Let $G$ be a finite abelian group. Let $G_{i}$ be subgroups of $G$ such that $\sum G_{i}=G$ and $\prod o\left(G_{i}\right)=o(G)$. Then $G=\oplus G_{i}$.

In [3, Proposition 2.6] we showed that the sum $\sum_{0<i \text { :odd }<k} G_{i}$ is not a direct sum if $u \neq 1$ and $a_{0} \leq \frac{k}{2}, a_{0} \neq 1$. The next theorem shows how to modify the generators of $G_{i}$ to get a direct sum decomposition of $U(R)$.

The cases $a=1,0$ being treated in [3], we will assume that $a_{0} \neq 1$ and $a_{0} \neq 0$.

Theorem 2.2. Let $R=\mathbb{Z}_{4}[X] /\left(X^{k}+2 t(X)\right)$, where $t(X)=X^{a_{0}}+\cdots+X^{a_{s}}$ with $a_{0}<\cdots<a_{s}$ and $a_{s}<k$. Using the notations above assume $u \neq 1$ and $a_{0} \leq \frac{k}{2}, a_{0} \neq 0,1$. Then there is $g_{i(s)}^{\prime} \in \sum_{k-a \leq j<k} G_{i(j)}$ with $k-a \leq i(s)<k$ such that

$$
U_{1}(R)=\bigoplus_{0<i: \text { odd }<k} G_{i}^{\prime} \oplus \bigoplus_{i \in S} H_{j}
$$

with

$$
G_{i}^{\prime}=\left\{\begin{array}{l}
\left\langle 1+X^{i}\right\rangle \text { with } l o\left(1+X^{i}\right)=k_{i}+1 \text { for } i \neq i(s), \\
\left\langle g_{i(s)}^{\prime}\right\rangle \text { with } l o\left(g_{i(s)}\right)=k_{i} \text { for } i=i(s)
\end{array}\right.
$$

and

$$
S=\{i \mid 0<i<a\} \cup\{a+i \mid 0<i \text { : odd }<k-2 a\} \cup\{k-a\} \text { if } k \text { is even. }
$$

Proof. By [3, Lemma 2.1], we know $\left\{i 2^{k_{i}-1} \mid 0<i\right.$ : odd $\left.<k\right\}=\left\{j \mid \frac{k}{2} \leq j<\right.$ $k$ \}. For each $j$ with $\frac{k}{2} \leq j<k$ let

$$
g_{j}(X)=\left(1+X^{i(j)}\right)^{2^{k_{i(j)}}}
$$

so that $j=i(j) 2^{k_{i(j)}}$. 
Hence if $j=i 2^{k_{i}}$, then

$$
\begin{aligned}
\left(1+X^{i}\right)^{2^{k_{i}}} & =1+2 X^{i 2^{k_{i}-1}}+X^{i 2^{k_{i}}} \\
& =1+2 X^{i 2^{k_{i}-1}}+2 u(X) X^{i 2^{k_{i}}-k+a} \\
& =1+2 X^{j}+2 u X^{2 j-k+a}
\end{aligned}
$$

which is a generator of $G_{i} \cap T$ which is cyclic of order 2 . Hence we see that the coefficients of $g_{j}^{0}=\left(1+X^{i}\right)^{2^{k_{i}}}-1$ for $0<i$ odd $<k$ looks like

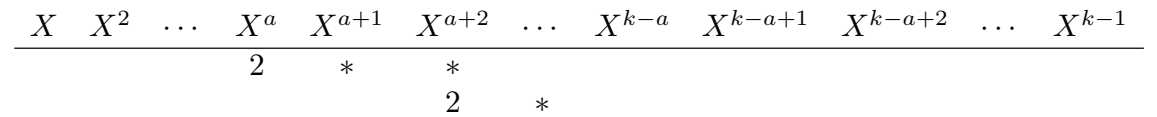

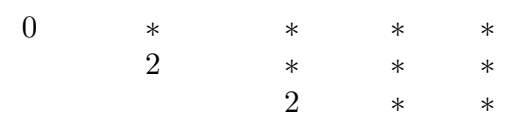

$$
\begin{aligned}
& 2
\end{aligned}
$$

The matrix above shows that the row containing 0 is a linear combination of the rows below. But this means that

$$
\left(1+X^{i(k-a)}\right)^{2^{k_{i(k-a)}}}=\prod_{j>k-a}\left(1+X^{i(j)}\right)^{2^{k_{i(j)}}} .
$$

Let $k_{i(s)}$ be the minimum of $k_{i(j)}$ 's which appear as the exponents in the equality above. Then

$$
\begin{aligned}
& {\left[\left(1+X^{i(k-a)}\right)^{2^{k_{i(k-a)}-k_{i(s)}}}\right]^{2^{k_{i(s)}}} } \\
= & \left(1+X^{i(s)}\right)^{2^{k_{i(s)}}} \prod_{k-a<j \neq s<k}\left[\left(1+X^{i(j)}\right)^{2^{k_{i(j)}-k_{i(s)}}}\right]^{2^{k_{i(s)}}} .
\end{aligned}
$$

Let

$$
g_{i(s)}^{\prime}=\left\{\begin{array}{cc}
\left(1+X^{i(s)}\right) \prod_{k-a<j \neq s<k}\left[\left(1+X^{i(j)}\right)^{\left.2^{k_{i(j)}-k_{i(s)}}\right](1+}\right. & \left.X^{i(k-a)}\right)^{-2^{k_{i(k-a)}-k_{i(s)}}} \\
& \text { if } s \neq k-a, \\
\left(1+X^{i(k-a)}\right) \prod_{k-a<j<k}\left(1+X^{i(j)}\right)^{-2^{\left.k_{i(j)}-k_{i(k-a)}\right)}} & \text { if } s=k-a,
\end{array}\right.
$$

and let $g_{i}^{\prime}=g_{i}$ for $i \neq i(s)$. If we let $G_{i}^{\prime}=\left\langle g_{i}^{\prime}\right\rangle$, then the consideration above shows that

$$
G_{i}^{\prime} \cap T=\left\{\begin{array}{l}
\left\langle\left(1+X^{i}\right)^{2^{k_{i}}}\right\rangle \text { if } 0<i: \text { odd }<k, i \neq i(s), \\
(1) \text { if } i=i(s)
\end{array}\right.
$$

which means, the matrix formed by the coefficients of $\left(g_{i}^{\prime}\right)^{0}$ is the same as the above except that all the entries of the $s$-th row $(k-a \leq s<k)$ are made to be 0 (by using elementary row operations). 
Let

$\mathcal{F}=\left\{1+2 X^{i} \mid 0<i<a\right\} \cup\left\{1+2 X^{a+i} \mid 0<i\right.$ : odd $\left.<k-2 a\right\} \cup\left\{1+2 X^{k-a}\right\}$.

Let $M$ be the matrix formed by the coefficients of $g_{i}^{0}$ with $0<i$ : odd $<k$ together with $f_{i}^{0}\left(f_{i} \in \mathfrak{F}\right)$; and $M^{\prime}$ be the matrix formed by the coefficients of $\left(g_{i}^{\prime}\right)^{0}$ together with $f_{i}^{0}\left(f_{i} \in \mathcal{F}\right)$. Then the subgroup of $2 \mathbb{Z}_{4}^{k-1}$ generated by the rows of $M$ is the same as the subgroup generated by the rows of $M^{\prime}$ since $M^{\prime}$ is obtained by performing elementary row operations on $M$. Now it is clear that the subgroup generated by the rows of $M$ is the whole group $2 \mathbb{Z}_{4}^{k-1}$. Therefore if we let $G^{\prime}=\sum G_{i}^{\prime}$, then $G^{\prime} \cap T_{1}$ together with $\mathcal{F}$ generate $T_{1}$. And obviously, $\phi\left(G^{\prime}\right)$ generate $U\left(\mathbb{F}_{2}[X] /\left(X^{k}\right)\right)$.

Finally we need to check that $\sum_{0<i \text { :odd }<k} G_{i}^{\prime}+\sum_{i \in S} l o\left(H_{i}\right)$ is a right number. But the situation is the same as [3, Theorem 3.4(ii)] and we skip our computation.

Example 2.3. Let $R=\mathbb{Z}_{4}[X] /\left(X^{5}+2 u X^{2}\right)$, where $u(X)=1+X+X^{2}$. Then $l o\left(U_{1}(R)\right)=8$. We observe $X^{8}=2 u X^{5}=0$. Then $l o(1+X)=4$, $l o\left(1+X^{3}\right)=2$. Further

$$
\begin{aligned}
(1+X)^{8} & =1+2 X^{4}, \\
\left(1+X^{3}\right)^{2} & =1+2 X^{3}+X^{6} \\
& =1+2 X^{3}+2 X^{3}\left(1+X+X^{2}\right) \\
& =1+2 X^{4} .
\end{aligned}
$$

Then $G_{1} \cap G_{3}=\left\langle 1+2 X^{4}\right\rangle$ and $\left(1+X^{3}\right)^{2}=\left((1+X)^{4}\right)^{2}$. And if we take

$$
\begin{aligned}
& G_{1}^{\prime}=G_{1}, \\
& G_{3}^{\prime}=\left\langle\left(1+X^{3}\right)(1+X)^{-4}\right\rangle,
\end{aligned}
$$

then $G_{1}^{\prime} \cap G_{3}^{\prime}=1$ and $\operatorname{lo}\left(G_{3}^{\prime}\right)=1$.

If we let $H_{1}=\langle 1+2 X\rangle, H_{2}=\left\langle 1+2 X^{2}\right\rangle, H_{1}=\left\langle 1+2 X^{3}\right\rangle$, then

$$
U(R)=G_{1}^{\prime} \oplus G_{3}^{\prime} \oplus H_{1} \oplus H_{2} \oplus H_{3} .
$$

Example 2.4. Let $R=\mathbb{Z}_{4}[X] /\left(X^{10}+2 u X^{4}\right)$, where $u(X)=1+X+X^{3}$. Then $l o\left(U_{1}(R)\right)=18$. We observe $X^{2 k-4}=X^{16}=2 u X^{10}=0$ and $k-a=$ $6=3 \cdot 2^{k_{3}-1}$. Now we compute

\begin{tabular}{|c|c|c|c|c|}
\hline $1+X^{i}$ & $k_{i}+1$ & $l o\left(G_{i}\right)$ & $\left(1+X^{i}\right)^{2^{k_{i}}}$ & \\
\hline $1+X$ & 5 & 5 & $(1+X)^{2^{4}}=1+2 X^{8}$ & 8 \\
$1+X^{3}$ & 3 & 3 & $\left(1+X^{3}\right)^{4}=1+2 X^{7}+2 X^{9}$ & $6=k-a=3 \cdot 2^{k_{3}-1}$ \\
$1+X^{5}$ & 2 & 2 & $\left(1+X^{5}\right)^{2}=1+2 X^{4}+2 X^{5}+2 X^{7}$ & 4 \\
$1+X^{7}$ & 2 & 2 & $\left(1+X^{7}\right)^{2}=1+2 X^{7}+2 X^{8}+2 X^{9}$ & 7 \\
$1+X^{9}$ & 2 & 2 & $\left(1+X^{9}\right)^{2}=1+2 X^{9}$ & 9 \\
\hline
\end{tabular}

The coefficients of $\left(1+X^{i}\right)^{2^{k_{i}}}-1$ looks like; 


\begin{tabular}{|c|c|c|c|c|c|c|c|c|}
\hline$X \quad X^{2}$ & $X^{3}$ & $X^{4}$ & $X^{5}$ & $X^{6}$ & $X^{7}$ & $X^{8}$ & $X^{9}$ & \\
\hline & & 2 & 0 & 0 & 0 & 0 & 0 & $\left(1+X^{5}\right)^{2}$ \\
\hline & & & & 0 & 2 & 0 & 2 & $\left(1+X^{3}\right)^{4}$ \\
\hline & & & & & 2 & 2 & 2 & $\left(1+X^{7}\right)^{2}$ \\
\hline & & & & & & 2 & 0 & $(1+X)^{16}$ \\
\hline & & & & & & & 2 & $\left(1+X^{9}\right)^{2}$ \\
\hline
\end{tabular}

The line joining the coefficient 2 of $X^{4}$ and 0 of $X^{6}$ has slope $-\frac{1}{2}$ and the line joining the coefficient 0 of $X^{6}$ and 2 of the coefficient of $X^{9}$ has slope -1 and the slope changes at the coefficient 0 of $X^{6}$.

Hence we see that

$$
\begin{aligned}
\left(1+X^{3}\right)^{4} & =1+2 X^{7}+2 X^{9} \\
& =\left(1+2 X^{7}+2 X^{8}+2 X^{9}\right)\left(1+2 X^{8}\right)=\left(1+X^{7}\right)^{2}(1+X)^{16} .
\end{aligned}
$$

Now let

$$
\begin{array}{ll}
g_{1}^{\prime}=1+X & \operatorname{lo}\left(g_{1}^{\prime}\right)=5 \\
g_{3}^{\prime}=1+X^{3} & l o\left(g_{3}^{\prime}\right)=3 \\
g_{5}^{\prime}=1+X^{5} & l o\left(g_{5}^{\prime}\right)=2 \\
g_{7}^{\prime}=\left(1+X^{7}\right)\left(1+X^{3}\right)^{-2}(1+X)^{-8} & l o\left(g_{7}^{\prime}\right)=1 \\
g_{9}^{\prime}=1+X^{9} & l o\left(g_{9}^{\prime}\right)=2 .
\end{array}
$$

Let $G_{i}^{\prime}=\left\langle g_{i}^{\prime}\right\rangle$. Then $l o\left(G_{i}^{\prime}\right)=k_{i}+1$ for $i \neq 7$ and $l o\left(G_{7}^{\prime}\right)=1$. Hence $\sum l o\left(G_{i}^{\prime}\right)=5+3+2+1+2=13$. Note also that $G_{7}^{\prime} \cap T=(1)\left(g_{7}^{\prime}\right.$ is of order 2 and $\left.g_{7}^{\prime} \notin \operatorname{Ker}(\phi)\right)$. Further, $G^{\prime} \cap T$ together with $\left\{1+2 X, 1+2 X^{2}, 1+2 X^{3}, 1+\right.$ $\left.2 X^{5}, 1+2 X^{6}\right\}$ generate $T$ and $\sum_{i=1,2,3,5,6} l o\left(H_{i}\right)=5$. Hence we conclude that

$$
U_{1}(R)=\bigoplus_{0<i: \text { odd }<10} G_{i}^{\prime} \oplus \bigoplus_{i=1,2,3,4,6} H_{i}
$$

\section{Decomposing the group of units of $\mathbb{Z}_{4}[X] /\left(X^{k}+2 u X^{a}, 2 X^{r}\right)$}

Throughout this section we let $R=\mathbb{Z}_{4}[X] /\left(X^{k}+2 u(X) X^{a}, 2 X^{r}\right)$, where $0 \leq a<r<k$ and $u(X)=1+X^{b_{1}}+\cdots+X^{b_{s}}$ with $a+s<r$. Let $t(X)=X^{a_{0}}+\cdots+X^{a_{s}}$ with $a_{0}<\cdots<a_{s}, a_{0}=a$ and $b_{i}+a=a_{i}$ for $0 \leq i \leq s$ so that $t(X)=X^{a} u(X)$.

Lemma 3.1. Let $R=\mathbb{Z}_{4}[X] /\left(X^{k}+2 u(X) X^{a}, 2 X^{r}\right)$. Then the number of elements of $U(R)$ is $2^{k+r-1}$ and the group of 1-units $U_{1}(R)$ has order $2^{k+r-2}$.

Proof. The proof is the same as [1, Lemma 6.1].

Lemma 3.2. Let $R=\mathbb{Z}_{4}[X] /\left(X^{k}+2 u X^{a}, 2 X^{r}\right)$, where $u(X)=1+X^{b_{1}}+$ $\cdots+X^{b_{s}}$. Suppose $k \geq r+a$ and let $\alpha=k+r-a$ and $\alpha_{i}=\left\lfloor\frac{k+r-a}{i}\right\rfloor_{2}$. Then lo $\left(1+X^{i}\right)=\alpha_{i}$ for all odd integer $i(0<i<k)$. 
Proof. We know that $\left(1+X^{i}\right)^{2^{\alpha_{i}}}=1$. We need to check whether $(1+$ $\left.X^{i}\right)^{2^{\alpha_{i}-1}}=1$. By [3, Lemma 2.1], we see that $\left\{i 2^{a_{i}-1} \mid 0<i\right.$ : odd $\left.<\alpha\right\}=$ $\left\{j \mid \frac{\alpha}{2} \leq j<\alpha\right\}$. Now

$$
\begin{aligned}
& \left(1+X^{i}\right)^{2^{\alpha_{i}-1}} \\
= & 1+2 X^{i 2^{\alpha_{i}-2}}+X^{i 2^{\alpha_{i}}} \\
= & 1+2 X^{i 2^{\alpha_{i}-2}}+2\left(X^{i 2^{\alpha_{i}-1}-k+a}+X^{i 2^{\alpha_{i}-1}-k+a+b_{1}}+\cdots+X^{i 2^{\alpha_{i}-1}-k+a+b_{s}}\right)
\end{aligned}
$$

only if $j=i 2^{\alpha_{i}} \geq k$. (Otherwise $\left(1+X^{i}\right)^{2^{\alpha_{i}}} \neq 1$.)

On the other hand, $k \geq r+a$ implies that $a<\frac{k}{2}$. Therefore $k-a>\frac{k}{2} \geq \frac{j}{2}$. Hence $\frac{j}{2}>j-k+a$ and consequently $\left(1+X^{i}\right)^{2^{\alpha_{i}-1}}=1+2 X^{i 2^{\alpha_{i}-1}-k+a}+(\mathrm{hdt}) \neq$ 1. Thus $l o\left(1+X^{i}\right)=\alpha_{i}$.

Remark. Note that if we let $\alpha_{i}=\left\lfloor\frac{k+r-a}{i}\right\rfloor_{2}$, then since $k<k+r-a<2 k$ we have

$$
k_{i} \leq \alpha_{i} \leq k_{i}+1
$$

Theorem 3.3. Let $R=\mathbb{Z}_{4}[X] /\left(X^{k}+2 u X^{a}, 2 X^{r}\right)$, where $u(X)=1+X^{b_{1}}+$ $\cdots+X^{b_{s}}$ with $0<a<r<k$. If $k \geq r+a$, then the group of 1-units $U_{1}(R)$ decomposes into the direct sum:

$$
U_{1}(R)=\bigoplus_{1 \leq i: \mathrm{odd}<k} G_{i} \oplus \bigoplus_{i \in S} H_{i},
$$

where $S=\{i \mid 0<i<a\} \cup\{a+l \mid l$ : odd $>0, a+l<r\}$. Here, $G_{i}$ is the cyclic subgroup generated by $1+X^{i}$ of order $2^{\alpha_{i}}$ and $H_{i}$ is the cyclic subgroup generated by $1+2 X^{i}$ of order 2 .

Proof. First we look at $G \cap T$. Since the elements of $T$ are order 2, the only possible elements in $G_{i}$ which are in $T$ are of the form $\left(1+X^{i}\right)^{\alpha_{i}-1}=1+$ $2 X^{i 2^{\alpha_{i}-2}}+X^{i 2^{\alpha_{1}-1}}$. This will belong to $T$ only if $i 2^{\alpha_{i}-1} \geq k$. Now $\left\{i 2^{\alpha_{i}-1} \mid 1 \leq\right.$ $i$ : odd $<\alpha\}=\left\{j \mid \frac{\alpha}{2} \leq j<\alpha\right\}$, by Lemma 2.1, which we will call $\mathfrak{S}$. If $j$ is odd such that $j \geq k$, then $\alpha_{j}=1$ and $\left(1+X^{j}\right)^{\alpha_{j}-1}=\left(1+X^{j}\right) \notin T$. Hence the even numbers $\geq k$ in $\mathfrak{S}$ is of the form $S^{\prime}=\left\{i 2^{\alpha_{i}-1} \mid i\right.$ : odd, $\alpha_{i}>$ $\left.1, i 2^{\alpha_{i}-1} \geq k\right\}=\{j$ : even $\mid k \leq j<\alpha\}$. Thence $G \cap T$ is generated by $\left\{1+2 X^{\frac{j}{2}}+2 u(X) X^{j-k+a} \mid j \in S^{\prime}\right\}$.

As in the proof of [3, Theorem 4.1], $\frac{j}{2}>j-k+a$ and hence $G \cap T$ is generated by $\left\{1+2 X^{a+2 i}+(\mathrm{hdt}) \mid i=0,1, \ldots\right\}$. Therefore we are reduced to the situation of the proof of [3, Theorem 4.1] and we safely omit the proof.

Example 3.4. Let $R=\mathbb{Z}_{4}[X] /\left(X^{20}+2 u(X) X^{5}, 2 X^{12}\right)$, where $u(X)=1+$ $X^{2}+X^{3}$. Here we have $k+r-a=27, k \geq r+a$ and $l o\left(U_{1}(R)\right)=k+r-2=30$. Also $X^{27}=2 u X^{12}=0$. Let $\alpha=k+r-a=27$ and $\alpha_{i}=\left\lfloor\frac{\alpha}{i}\right\rfloor_{2}=\left\lfloor\frac{27}{i}\right\rfloor_{2}$.

We look at the possible elements of $T$ of the form $\left(1+X^{i}\right)^{2^{\alpha_{i}-1}}$. 


\begin{tabular}{|c|c|c|c|c|}
\hline $1 \leq i:$ odd $<\alpha$ & $\alpha_{i}$ & $i 2^{\alpha_{i}-1}$ & $\left(1+X^{i}\right)^{\alpha^{\alpha^{-1}}}$ & \\
\hline 1 & 5 & $2^{4}=16$ & $1+2 X^{8}+X^{16}$ & not in $T$ \\
3 & 4 & $3 \cdot 2^{3}=24$ & $1+2\left(X^{9}+X^{11}\right)$ & \\
5 & 3 & $5 \cdot 2^{2}=20$ & $1+2 X^{10}+2\left(X^{5}+X^{7}+X^{8}\right)$ & \\
7 & 2 & $7 \cdot 2=14$ & $1+2 X^{7}+X^{14}$ & not in $T$ \\
9 & 2 & $9 \cdot 2=18$ & $1+2 X^{9}+X^{18}$ & not in $T$ \\
11 & 2 & $11 \cdot 2=22$ & $1+2 X^{11}+2\left(X^{7}+X^{9}+X^{10}\right)$ & \\
13 & 2 & $13 \cdot 2=26$ & $1+2 X^{11}$ & not in $T$ \\
15 & 1 & 15 & $1+X^{15}$ & not in $T$ \\
17 & 1 & 17 & $1+X^{17}$ & not in $T$ \\
19 & 1 & 19 & $1+X^{19}$ & \\
21 & & 21 & & \\
23 & & 23 & & \\
25 & & 25 & & \\
27 & & 27 & & \\
29 & & 29 & & \\
\hline
\end{tabular}

Now we list the coefficients of $\left(1+X^{i}\right)^{2^{\alpha_{i}}}-1$;

\begin{tabular}{|c|c|c|c|c|c|c|c|}
\hline$X^{5}$ & $X^{6}$ & $X^{7}$ & $X^{8}$ & $X^{9}$ & $X^{10}$ & $X^{11}$ & $\left(1+X^{i}\right)^{2^{\alpha_{i}}}$ \\
\hline \multirow[t]{4}{*}{2} & & 2 & 2 & & 2 & & $\left(1+X^{5}\right)^{4}$ \\
\hline & & 2 & & 2 & 2 & 2 & $\left(1+X^{11}\right)^{2}$ \\
\hline & & & & 2 & & 2 & $\left(1+X^{3}\right)^{8}$ \\
\hline & & & & & & 2 & $\left(1+X^{13}\right)^{2}$ \\
\hline
\end{tabular}

Therefore $T \cap G$ together with $\left\{1+2 X, 1+2 X^{3}, 1+2 X^{5}, 1+2 X^{7}, 1+2 X^{9}, 1+\right.$ $\left.2 X^{11}\right\}$ generate $T$. Of course $\phi(G)$ generate $U\left(\mathbb{F}_{2}[X] /\left(X^{k}\right)\right)$.

Now

$$
\sum_{1 \leq i: \text { odd }<20} l o\left(G_{i}\right)=\sum_{1 \leq i: \text { odd }<k} \alpha_{i}=5+4+\cdots+1=24,
$$

and

$$
\sum\left\{l o\left(H_{i}\right) \mid i=1,3,5,7,9,11\right\}=6 .
$$

Hence

$$
U_{1}(R)=\bigoplus_{1 \leq i \text { odd }<20} G_{i} \oplus \bigoplus\left\{H_{i} \mid i=1,3,5,7,9,11\right\},
$$

where $G_{i}$ are cyclic generated by $1+X^{i}$ for each odd $i$ with $l o\left(G_{i}\right)=\alpha_{i} ; H_{i}$ are cyclic with $l o\left(H_{i}\right)=1$ generated by $1+2 X^{i}$.

Lemma 3.5. Let $R=\mathbb{Z}_{4}[X] /\left(X^{k}+2 u X^{a}, 2 X^{r}\right)$, where $u(X)=1+X^{b_{1}}+$ $\cdots+X^{b_{s}}$. Suppose $k<r+a$ and $a>\frac{k}{2}$. Then lo $\left(1+X^{i}\right)=r_{i}+1$ for all odd integer $i(0<i<k)$.

Proof. We know that $\left(1+X^{i}\right)^{2^{r_{i}+1}}=1$. We need to check whether $\left(1+X^{i}\right)^{2^{r_{i}}}=$ 1. By [3, Lemma 2.1], we see that $\left\{i 2^{r_{i}} \mid 0<i\right.$ : odd $\left.<2 r\right\}=\{j \mid r \leq j<2 r\}$. 
Now

$$
\begin{aligned}
& \left(1+X^{i}\right)^{2^{r_{i}}} \\
= & 1+2 X^{i 2^{r_{i}-1}}+X^{i 2^{r_{i}}} \\
= & 1+2 X^{i 2^{r_{i}-1}}+2\left(X^{i 2^{r_{i}}-k+a}+X^{i 2^{r_{i}}-k+a+b_{1}}+\cdots+X^{i 2^{r_{i}}-k+a+b_{s}}\right)
\end{aligned}
$$

only if $j=i 2^{r_{i}} \geq k$. (Otherwise $\left(1+X^{i}\right)^{2^{r_{i}}} \neq 1$.) On the other hand, $a>\frac{k}{2}$ together with $j=i 2^{r_{i}} \geq k$ implies that $\frac{j}{2}<j-k+a$. Therefore $\left(1+X^{i}\right)^{2^{r_{i}}}=1+2 X^{i 2^{r_{i}-1}}+(\mathrm{hdt}) \neq 1$. Hence $l o\left(1+X^{i}\right)=r_{i}+1$.

Remark. Note that since $k \leq r+a \leq 2 r \leq 2 k$ we have

$$
k_{i} \leq\left\lfloor\frac{2 r}{i}\right\rfloor_{2} \leq k_{i}+1
$$

Theorem 3.6. Let $R=\mathbb{Z}_{4}[X] /\left(X^{k}+2 u X^{a}, 2 X^{r}\right)$, where $u(X)=1+X^{b_{1}}+$ $\cdots+X^{b_{s}}$ with $0<a<r<k$. If $k<r+a$ and $a>\frac{k}{2}$, then the group of 1-units $U_{1}(R)$ decomposes into the direct sum:

$$
U_{1}(R)=\bigoplus_{0<i: \text { odd }<k} G_{i} \oplus \bigoplus_{0<i<\frac{k}{2}} H_{i}
$$

where $G_{i}$ is cyclic generated by $1+X^{i}$ with $l o\left(G_{i}\right)=r_{i}+1$ and $H_{i}$ is cyclic generated by $1+2 X^{i}$ with $l o\left(H_{i}\right)=1$.

Proof. As before consider the exact sequence

$$
(1) \rightarrow T \rightarrow U_{1}\left(\mathbb{Z}_{4}[X] /\left(X^{k}+2 X^{a}, 2 X^{r}\right)\right) \stackrel{\phi}{\rightarrow} U\left(\mathbb{F}_{2}[X] /\left(X^{k}\right)\right) \rightarrow(1),
$$

where $T$ is generated by $1+2 X^{i}$ where $0<i<r$. As usual we need to show that $G \cap T$ together with $\bigoplus_{0<i<\frac{k}{2}} H_{i}$ generate $T$ when $a>\frac{k}{2}$.

By Lemma 2.1, we see that

$$
\left\{i 2^{r_{i}} \mid 0<i \text { : odd }<2 r\right\}=\{j \mid r \leq j<2 r\}
$$

which we call $\mathfrak{R}$. If $i \in \mathfrak{R}$ is odd such that $i \geq k$, then $r_{i}=0$ and hence $\left(1+X^{i}\right)^{2^{r_{i}}} \notin T$. And therefore if $n \in \mathfrak{R}$ is even such that $k \leq n<2 r$, then it is of the form $n=i 2^{r_{i}}$ with $r_{i} \geq 1$ and $i$ is odd such that $0<i<2 r$ (we only need $i$ for which $0<i<k$ ). For an odd $i$ with $k \leq j=i 2^{r_{i}}$ and $0<i<k$ we have

$$
\begin{aligned}
\left(1+X^{i}\right)^{2^{r_{i}}} & =1+2 X^{i 2^{r_{i}-1}}+X^{i 2^{r_{i}}} \\
& =1+2 X^{i 2^{r_{i}-1}}+2 u X^{i 2^{r_{i}}-k+a} \in T .
\end{aligned}
$$

Since $\frac{k}{2}<a$ we see that $k-a<\frac{k}{2}$ and as $k \leq n$ we conclude that $k-a<\frac{n}{2}$. This in turn implies that $n-k+a>\frac{n}{2}$. Thus $\left(1+X^{i}\right)^{2^{r_{i}}}=1+2 X^{\frac{n}{2}}+$ (hdt) for all $n \geq \frac{k}{2}$.

Thus we conclude that $G \cap T$ together with $\left\{1+2 X^{i} \mid 0<i<\frac{k}{2}\right\}$ generate $T$ by Lemma 2.4 . 
To finish our proof we need to show that the sum of the logarithmic order of our subgroups is the right number. But this is the same as [3, Theorem 4.2].

Example 3.7. Let $R=\mathbb{Z}_{4}[X] /\left(X^{20}+2 u X^{11}, 2 X^{17}\right)$, where $u(X)=1+X^{2}+$ $X^{3}$. Here we have $r+a=28, k<r+a$ and $a=11>10=\frac{k}{2}$. Also $l o\left(U_{1}(R)\right)=k+r-2=35$ and $X^{26}=2 u X^{17}=0$. Let $r_{i}=\left\lfloor\frac{r}{i}\right\rfloor_{2}$ so that $r_{i}+1=\left\lfloor\frac{2 r}{i}\right\rfloor_{2}$.

We look at the possible elements of $T$ of the form $\left(1+X^{i}\right)^{2^{r_{i}}}$.

\begin{tabular}{|c|c|c|c|c|}
\hline $1 \leq i:$ odd $<34$ & $r_{i}$ & $i 2^{r_{i}}$ & $\left(1+X^{i}\right)^{2^{r_{i}}}$ & \\
\hline 1 & 5 & 32 & $1+2 X^{16}$ & \\
3 & 3 & $3 \cdot 2^{3}=24$ & $1+2 X^{12}+2 u X^{15}$ & \\
5 & 2 & $5 \cdot 2^{2}=20$ & $1+2 X^{10}+2 u X^{11}$ & \\
7 & 2 & $7 \cdot 2^{2}=28$ & $1+2 X^{14}$ & \\
9 & 1 & $9 \cdot 2=18$ & $1+2 X^{9}+X^{18}$ & not in $T_{1}$ \\
11 & 1 & $11 \cdot 2=22$ & $1+2 X^{11}+2 u X^{13}$ & \\
13 & 1 & $13 \cdot 2=26$ & $1+2 X^{13}$ & \\
15 & 1 & $15 \cdot 2=30$ & $1+2 X^{15}$ & \\
17 & 0 & 17 & $1+X^{17}$ & not in $T_{1}$ \\
19 & 0 & 19 & $1+X^{19}$ & not in $T_{1}$ \\
21 & & 21 & & \\
23 & & 23 & & \\
25 & & 25 & & \\
27 & & 27 & & \\
29 & & 29 & & \\
31 & & 31 & & \\
33 & & 33 & & \\
\hline
\end{tabular}

Now we list the coefficients of $\left(1+X^{i}\right)^{2^{r_{i}}}$;

\begin{tabular}{ccccccc|c}
$X^{10}$ & $X^{11}$ & $X^{12}$ & $X^{13}$ & $X^{14}$ & $X^{15}$ & $X^{16}$ & $\left(1+X^{i}\right)^{\alpha^{\alpha_{i}}}$ \\
\hline 2 & 2 & & 2 & 2 & & & $\left(1+X^{5}\right)^{4}$ \\
& 2 & & 2 & & 2 & 2 & $\left(1+X^{11}\right)^{2}$ \\
& 2 & & & 2 & & $\left(1+X^{3}\right)^{8}$ \\
& & 2 & & & & $\left(1+X^{13}\right)^{2}$ \\
& & & 2 & & & $\left(1+X^{7}\right)^{2}$ \\
& & & & & 2 & & $\left(1+X^{15}\right)^{2}$ \\
& & & & & & 2 & $(1+X)^{32}$
\end{tabular}

Therefore $G \cap T$ together with $H_{i},(i=1,2, \ldots, 9)$ generate $T$.

Now $l o\left(G_{i}\right)=r_{i}+1$ and $\sum_{1 \leq i \text { :odd }<20} l o\left(G_{i}\right)=26$. Further, $\sum l o\left(G_{i}\right)+$ $\sum_{i=1}^{9} l o\left(H_{i}\right)=35$. Hence

$$
U_{1}(R)=\bigoplus_{1 \leq i \text { :odd }<20} G_{i} \oplus \bigoplus\left\{H_{i} \mid i=1,2, \ldots, 9\right\}
$$


where $G_{i}$ are cyclic generated by $1+X^{i}$ for each odd $i$ with $l o\left(G_{i}\right)=\alpha_{i} ; H_{i}$ are cyclic with $l o\left(H_{i}\right)=1$ generated by $1+2 X^{i}$.

Lemma 3.8. Let $R=\mathbb{Z}_{4}[X] /\left(X^{k}+2 u X^{a}, 2 X^{r}\right)$, where $u(X)=1+X^{b_{1}}+$ $\cdots+X^{b_{s}}$. Assume $k<a+r$ and $a \leq \frac{k}{2}$. Then there is an odd $i(0<i<k)$ with $k-a=i 2^{r_{i}-1}, r_{i} \geq 1$. For such an $i$ we have

$$
l o\left(1+X^{i}\right)= \begin{cases}r_{i}+1 & \text { if } i 2^{r_{i}-1}+b_{1}<r, \\ r_{i} & \text { if } i 2^{r_{i}-1}+b_{1} \geq r .\end{cases}
$$

Proof. By [3, Lemma 2.1], we see that $\left\{i 2^{r_{i}} \mid 0<i\right.$ : odd $\left.<2 r\right\}=\{j \mid r \leq$ $j<2 r\}$. If $j$ is even and $j \geq k$, then it is of the form $i 2^{r_{i}}$ with $r_{i} \geq 1$. Our conditions $k<a+r$ and $a \leq \frac{k}{2}$ imply that $k \leq 2(k-a)<2 r$. Hence there is an odd $i$ such that $2(k-a)=i 2^{r_{i}}$ with $r_{i} \geq 1$.

Now suppose $2(k-a)=i 2^{r_{i}}$ with $r_{i} \geq 1$. Then

$$
\begin{aligned}
& \left(1+X^{i}\right)^{2^{r_{i}}} \\
= & 1+2 X^{i 2^{r_{i}-1}}+X^{i 2^{r_{i}}} \\
= & 1+2 X^{i 2^{r_{i}-1}}+2\left(X^{i 2^{r_{i}}-k+a}+X^{i 2^{r_{i}}-k+a+b_{1}}+\cdots+X^{i 2^{r_{i}}-k+a+b_{s}}\right) \\
= & 1+2\left(X^{i 2^{r_{i}-1}+b_{1}}+\cdots+X^{i 2^{r_{i}-1}+b_{s}}\right) .
\end{aligned}
$$

Now it is clear that the logarithmic order of $1+X^{i}$ is as stated.

Theorem 3.9. Let $R=\mathbb{Z}_{4}[X] /\left(X^{k}+2 u X^{a}, 2 X^{r}\right)$, where $u(X)=1+X^{b_{1}}+$ $\cdots+X^{b_{s}}$ with $0<a<r<k$ and $u \neq 1$. If $k<r+a$ and $a \leq \frac{k}{2}$, then there is an odd integer $j(0<j<k)$ such that $k-a=j 2^{r_{j}-1}$ and

where

$$
U_{1}(R)=\bigoplus_{0<i: \text { odd }<k} G_{i} \oplus \bigoplus_{i<\in S} H_{i}
$$

$S=\left\{\begin{array}{l}\{0<i<a\} \cup\{a+l \mid 0<l: \text { odd }<k-2 a\} \cup\{k-a\} \text { when } k \text { is even, } \\ \{0<i \leq a\} \cup\{a+l \mid 1<l: \text { even }<k-2 a\} \cup\{k-a\} \text { when } k \text { is odd. }\end{array}\right.$

Here, $G_{i}$ is cyclic generated by $1+X^{i}$ with $l o\left(G_{i}\right)=r_{i}+1$ for $i \neq j$ and $l o\left(G_{j}\right)=r_{j}$; and $H_{i}$ is cyclic generated by $1+2 X^{i}$ with $l o\left(H_{i}\right)=1$.

Proof. The proof will be similar to the proof of Theorem 2.2. First we need to investigate $G \cap T$. By [3, Lemma 2.1] we see that $\left\{i 2^{r_{i}} \mid 0<i\right.$ : odd $\left.<2 r\right\}=$ $\{n \mid r \leq n<2 r\}$ which we call $\mathfrak{R}$. We define $n(i)$ and $i(n)$ by

$$
n(i)=i 2^{r_{i}} \text { and } n=i(n) 2^{r_{i(n)}} .
$$

But our assumption implies that $r<k-a$. On the other hand, since $a<r$ we have $k<a+r<2 r$. Therefore we see $r<k-a<2 r$ which shows that there is $j$ such that $k-a=j 2^{r_{j}-1}$.

The possible elements of $G_{i} \cap T$ are $\left(1+X^{i}\right)^{2^{r_{i}}}$ with $i 2^{r_{i}} \geq k$. (It has to be order 2 and this is the only one of order 2 in $G_{i}$ and the inequality $i 2^{r_{i}} \geq k$ 
guarantees that it is in $\operatorname{Ker}(\phi)$.) If $n=i 2^{r_{i}} \in \mathfrak{R}$ is odd with $n \geq k$, then $r_{i}=0$. Hence $\left(1+X^{i}\right)^{2^{r_{i}}} \notin T$. Therefore $\left(1+X^{i}\right)^{2^{r_{i}}} \in T$ exactly when $i 2^{r_{i}} \geq k$ with $r_{i} \geq 1$ and $0<i$ : odd $<k$ which are precisely $\{n$ : even $\mid k \leq n<2 r\}$ which we will call $\mathfrak{R}^{\prime}$.

If $n \in \mathfrak{R}^{\prime}$ with $\frac{n}{2}<k-a$ (resp. $\frac{n}{2}=k-a$, resp. $\frac{n}{2}>k-a$ ), then $\frac{n}{2}>n-k+a$ (resp. $\frac{n}{2}=n-k+a$, resp. $\left.\frac{n}{2}<n-k+a\right)$. Hence $G \cap T$ is generated by the following elements

$$
\begin{cases}1+2\left(X^{n-k+a}+(\text { hdt })\right) & \text { when } k \leq n<2(k-a), \\ 1+2\left(X^{a_{1}}+(\text { hdt })\right) & \text { when } \frac{n}{2}=k-a \\ 1+2\left(X^{\frac{n}{2}}+(\text { hdt })\right) & \text { when } \frac{n}{2}>k-a .\end{cases}
$$

As in the proof of Theorem 2.2, the second elements is a linear combination of the elements if the third type. Let

$$
1+2\left(X^{a_{1}}+(\mathrm{hdt})\right)=\prod_{i}\left(1+2\left(X^{\frac{n(i)}{2}}+(\mathrm{hdt})\right)\right) .
$$

But this means that

$$
\left(1+X^{i\left(\frac{n}{2}\right)}\right)^{2^{r_{i\left(\frac{n}{2}\right)}}}=\prod_{\frac{n}{2}>k-a}\left(1+X^{i(n)}\right)^{2^{r_{i(n)}}} .
$$

Let $r_{i(s)}$ be the minimum of $r_{i}$ 's. Then

$$
\begin{aligned}
& {\left[\left(1+X^{i\left(\frac{n}{2}\right)}\right)^{2^{\left.r_{i\left(\frac{n}{2}\right.}\right)-r_{i}(s)}}\right]^{2^{r_{i(s)}}} } \\
= & \left(1+X^{i(s)}\right)^{2^{r_{i(s)}}} \prod_{\frac{n}{2}>k-a, n \neq s}\left[\left(1+X^{i(n)}\right)^{2^{k_{i(n)}-r_{i(s)}}}\right]^{2^{r_{i(s)}} .}
\end{aligned}
$$

Let

$$
g_{i(s)}^{\prime}= \begin{cases}\left(1+X^{i(s)}\right) \prod_{\frac{n}{2}>k-a, n \neq s}\left[\left(1+X^{i(n)}\right)^{\left.2^{r_{i(n)}-r_{i(s)}}\right]}\right]\left(1+X^{i\left(\frac{n}{2}\right)}\right)^{-2^{r_{i\left(\frac{n}{2}\right)}-r_{i(s)}}} & \text { if } s \neq 0, \\ \left(1+X^{i\left(\frac{n}{2}\right)}\right) \prod_{k-a<\frac{n}{2}<r}\left(1+X^{i(n)}\right)^{-2^{r_{i(n)}-r_{i\left(\frac{n}{2}\right)}}} & \text { if } s=0,\end{cases}
$$

and let $g_{i}^{\prime}=g_{i}$ for $i \neq i(s)$. If we let $G_{i}^{\prime}=\left\langle g_{i}^{\prime}\right\rangle$, then the consideration above shows that

$$
G_{i}^{\prime} \cap T_{1}=\left\{\begin{array}{l}
\left\langle\left(1+X^{i}\right)^{2^{r_{i}}}\right\rangle \text { if } 0<i: \text { odd }<k, i \neq i(s), \\
(1) \text { if } i=i(s) .
\end{array}\right.
$$

Let

$\mathcal{F}=\left\{1+2 X^{i} \mid 0<i<a\right\} \cup\left\{1+2 X^{a+l} \mid 0<l\right.$ : odd $\left.<k-2 a\right\} \cup\left\{1+2 X^{k-a}\right\}$ when $k$ is even; and

$\mathcal{F}^{\prime}=\left\{1+2 X^{i} \mid 0<i \leq a\right\} \cup\left\{1+2 X^{a+l} \mid 1<l:\right.$ even $\left.<k-2 a\right\} \cup\left\{1+2 X^{k-a}\right\}$ 
when $k$ is odd. As before if we let $G^{\prime}=\sum G_{i}^{\prime}$, then $G^{\prime} \cap T$ together with $\mathcal{F}$ generate $T$. And obviously, $\phi\left(G^{\prime}\right)$ generate $U\left(\mathbb{F}_{2}[X] /\left(X^{k}\right)\right)$.

Finally we need to check that $\sum_{0<i \text { :odd }<k} G_{i}^{\prime}+\sum_{i \in S} l o\left(H_{i}\right)$ is a right number. But this is similar to [3, Theorem $4.2(\mathrm{ii})]$ and we omit the proof safely.

Example 3.10. Let $R=\mathbb{Z}_{4}[X] /\left(X^{20}+2 u X^{7}, 2 X^{17}\right)$, where $u(X)=1+X^{2}+$ $X^{3}$. Then we have $X^{30}=2 u X^{17}=0$. Here we have $r+a=24, k \leq r+a$ and $7=a \leq \frac{k}{2}=10$; and $l o\left(U_{1}(R)\right)=k+r-2=35$. Let $r_{i}=\left\lfloor\frac{r}{i}\right\rfloor_{2}$ so that $r_{i}+1=\left\lfloor\frac{2 r}{i}\right\rfloor_{2}$.

We look at the possible elements of $T$ of the form $\left(1+X^{i}\right)^{2^{r_{i}}}$.

\begin{tabular}{|c|c|c|c|c|}
\hline $1 \leq i:$ odd $<34$ & $r_{i}$ & $i 2^{r_{i}}$ & $\left(1+X^{i}\right)^{2^{r_{i}}}$ & \\
\hline 1 & 5 & 32 & $1+2 X^{16}+2 u X^{19}$ & \\
3 & 3 & $3 \cdot 2^{3}=24$ & $1+2 X^{12}+2 u X^{11}$ & \\
5 & 2 & $5 \cdot 2^{2}=20$ & $1+2 X^{10}+2 u X^{7}$ & \\
7 & 2 & $7 \cdot 2^{2}=28$ & $1+2 X^{14}+2 u X^{15}$ & \\
9 & 1 & $9 \cdot 2=18$ & $1+2 X^{9}+X^{18}$ & not in $T$ \\
11 & 1 & $11 \cdot 2=22$ & $1+2 X^{11}+2 u X^{9}$ & \\
13 & 1 & $13 \cdot 2=26$ & $1+2 X^{13}+2 u X^{13}$ & \\
15 & 1 & $15 \cdot 2=30$ & $1+2 X^{15}+2 u X^{17}$ & \\
17 & 0 & 17 & $1+X^{17}$ & not in $T$ \\
19 & 0 & 19 & $1+X^{19}$ & not in $T$ \\
21 & & 21 & & \\
23 & & 23 & & \\
25 & & 25 & & \\
27 & & 27 & & \\
29 & & 29 & & \\
31 & & 31 & & \\
33 & & 33 & & \\
\hline
\end{tabular}

Now we list the coefficients of $\left(1+X^{i}\right)^{2^{r_{i}}}$;

\begin{tabular}{|c|c|c|c|c|c|c|c|c|c|c|}
\hline$X^{7}$ & $X^{8}$ & $X^{9}$ & $X^{10}$ & $X^{11}$ & $X^{12}$ & $X^{13}$ & $X^{14}$ & $X^{15}$ & $X^{16}$ & $\left(1+X^{i}\right)^{2^{\alpha_{i}}}$ \\
\hline \multirow[t]{7}{*}{2} & & 2 & & & & & & & & $\left(1+X^{5}\right)^{4}$ \\
\hline & & 2 & & & 2 & & & & & $\left(1+X^{11}\right)^{2}$ \\
\hline & & & & 2 & 2 & 2 & 2 & & & $\left(1+X^{3}\right)^{8}$ \\
\hline & & & & & & 0 & & 2 & 2 & $\left(1+X^{13}\right)^{2}$ \\
\hline & & & & & & & 2 & 2 & & $\left(1+X^{7}\right)^{4}$ \\
\hline & & & & & & & & 2 & & $\left(1+X^{15}\right)^{2}$ \\
\hline & & & & & & & & & 2 & $(1+X)^{32}$ \\
\hline
\end{tabular}

The table above shows that

$$
\left(1+X^{13}\right)^{2}=\left(1+X^{15}\right)^{2}(1+X)^{32} .
$$


Hence we let

$$
\left\{\begin{array}{l}
g_{i}^{\prime}=1+X^{i} \text { if } i \text { is odd }<k \\
g_{13}^{\prime}=\left(1+X^{13}\right)\left(1+X^{15}\right)^{-1}(1+X)^{-16},
\end{array}\right.
$$

and let $G_{i}^{\prime}=\left\langle g_{i}^{\prime}\right\rangle$. Then

$$
\left\{\begin{array}{l}
l o\left(G_{i}^{\prime}\right)=r_{i}+1 \text { if } i \text { is odd }<k, \\
l o\left(G_{13}^{\prime}\right)=r_{i}
\end{array}\right.
$$

and $G_{13}^{\prime} \cap T=(1)$. If we let $G^{\prime}=\oplus G_{i}^{\prime}$, then it is easy to show that $\phi\left(G_{i}^{\prime}\right)$ generate $U_{1}\left(\mathbb{F}_{2}[X] /\left(X^{k}\right)\right)$. Further, the table above shows that $G^{\prime} \cap T$ together with

$$
H_{S}=\bigoplus_{i \in S} H_{i}=\bigoplus_{i \in S}\left\{1+2 X^{i}\right\}, \quad \text { where } S=\{1,2,3,4,5,6 ; 8,10,12 ; 13\}
$$

generate $T$. Now $\sum l o\left(G_{i}^{\prime}\right)=25$ and $l o\left(H_{S}\right)=10$.

Hence

$$
U_{1}(R)=\bigoplus_{1 \leq i: \text { odd }<20} G_{i}^{\prime} \oplus \bigoplus_{i \in S} H_{i}
$$

where $G_{i}$ are cyclic generated by $1+X^{i}$ for each odd $i ; H_{i}$ are cyclic with $l o\left(H_{i}\right)=1$ generated by $1+2 X^{i}$ for $i \in S$.

Theorem 3.11. Let $R=\mathbb{Z}_{4}[X] /\left(X^{k}+2 u(X), 2 X^{r}\right)$ where $u(X)=1+X^{b_{1}}+$ $\cdots+X^{b_{s}}$. Then the group of units $U(R)$ of $R$ is isomorphic to

$$
U(R)=\bigoplus_{1 \leq i: \text { odd }<k} G_{i} \oplus \bigoplus_{i \in S} H_{i}
$$

where

$$
S=\left\{\begin{array}{l}
\{i \text { : even } \mid 0 \leq i<r\} \text { if } k \text { is odd, } \\
\{i \text { : odd } \mid 0<i<r\} \cup\{0\} \text { if } k \text { is even. }
\end{array}\right.
$$

Here, $G_{i}$ is the cyclic group generated by $1+X^{i}$ with $l o\left(G_{i}\right)=\alpha_{i}$, where $\alpha=k+r$ and $\alpha_{i}=\left\lfloor\frac{\alpha}{i}\right\rfloor_{2}$; and $H_{i}$ is cyclic generated by $1+2 X^{i}$ with $l o\left(H_{i}\right)=1$.

Proof. By the exact sequence

$$
(1) \rightarrow T_{0} \rightarrow U\left(\mathbb{Z}_{4}[X] /\left(X^{k}+2 u(X), 2 X^{r}\right)\right) \stackrel{\phi}{\rightarrow} U\left(\mathbb{F}_{2}[X] /\left(X^{k}\right)\right) \rightarrow(1),
$$

where $T_{0}=\sum_{0 \leq i<r} H_{i}$, we need to show that $\left\{1+2 X^{i} \mid i \in S\right\}$ together with $G \cap T_{0}$ with odd $i$ generate $T_{0}$ and the sum of our subgroups has the right order (Recall $H_{0}=\langle-1\rangle$.)

First we look at $G \cap T_{0}$. Let $\alpha=k+r$ and $\alpha_{i}=\left\lfloor\frac{\alpha}{i}\right\rfloor_{2}$. Since the elements of $T_{0}$ are of order 2, the only possible elements in $G$ which are in $T_{0}$ are of the form $\left(1+X^{i}\right)^{\alpha_{i}-1}=1+2 X^{i 2^{\alpha_{i}-2}}+X^{i 2^{\alpha_{1}-1}}$ which belong to $T_{0}$ only if $i 2^{\alpha_{i}-1} \geq k$. Now we know that $\left\{i 2^{\alpha_{i}-1} \mid 1 \leq i\right.$ : odd $\left.<\alpha\right\}=\left\{j \mid \frac{\alpha}{2} \leq j<\alpha\right\}$ which we will call $\mathfrak{S}$. If $i$ is odd such that $i \geq k$, then $\alpha_{i}=1 ; i 2^{\alpha_{i}-1}=i$ and $\left(1+X^{i}\right)^{\alpha_{i}-1}=\left(1+X^{i}\right) \notin T_{0}$. Therefore the even numbers $\geq k$ in $\mathfrak{S}$ 
is of the form $S^{\prime}=\left\{i 2^{\alpha_{i}-1} \mid i\right.$ : odd, $\left.\alpha_{i}>1, i 2^{\alpha_{i}-1} \geq k\right\}=\{j$ : even $\mid k \leq$ $j<\alpha\}$. Since $j<2 k$ for $j \in S$ we have $\frac{j}{2}>j-k$. And hence $G \cap T_{0}=$ $\left\{1+2 X^{j-k} u(X)+2 X^{\frac{j}{2}} \mid j \in S^{\prime}\right\}$. Now by [3, Lemma 2.4] we see that $G \cap T_{0}$ together with $\left\{1+2 X^{i} \mid i \in S\right\}$ generate $T_{0}$.

As we already know that $\phi_{2}(G)$ generate $\mathbb{F}_{2}[X] /\left(X^{k}\right)$ we conclude that $G$ together with $\oplus_{i \in S} H_{i}$ generate $U(R)$.

Now we need to show that $\sum_{0<i \text { :odd }<k} l o\left(G_{i}\right)+\sum_{i \in S} l o\left(H_{i}\right)=k+r-1$. But this is the same as [3, Theorem 4.2].

\section{References}

[1] B. R. McDonald, Finite Rings with Identity, Pure and Applied Mathematics, Vol. 28. Marcel Dekker, Inc., New York, 1974.

[2] S. S. Woo, The group of units of some finite local rings I, J. Korean Math. Soc. 46 (2009), no. 2, 295-311.

[3] _ The group of units of some finite local rings II, J. Korean Math. Soc. 46 (2009), no. $3,475-491$.

Department of Mathematics

Ewha Women's University

SeOul 120-750, Korea

E-mail address: sswoo@ewha.ac.kr 